\title{
Lean Mass Loss and Altered Muscular Aerobic Capacity after Bariatric Surgery
}

\author{
Na Zhou Corentin Scoubeau $^{\mathrm{a}, \mathrm{b}}$ Kevin Forton ${ }^{\mathrm{a}, \mathrm{c}}$ Patricia Loi ${ }^{\mathrm{d}}$ Jean Closset ${ }^{\mathrm{d}}$ \\ Gael Deboeck $^{\mathrm{e}}$ Jean-Jacques Moraine ${ }^{a} \quad$ Malgorzata Klass $^{b, f}$ Vitalie Faoro $^{a}$ \\ ${ }^{a}$ Cardio-Pulmonary Exercise Laboratory, Faculty of Motor Sciences, Université Libre de Bruxelles, Brussels, Belgium; \\ bLaboratory for Biometry and Exercise Nutrition, Faculty of Motor Sciences, Université Libre de Bruxelles, Brussels, \\ Belgium; 'Department of Cardiology, Erasmus Hospital, Brussels, Belgium; dDepartment of Gastric Surgery, Erasmus \\ Hospital, Brussels, Belgium; 'Laboratory of Rehabilitation, Faculty of Motor Sciences, Université Libre de Bruxelles,

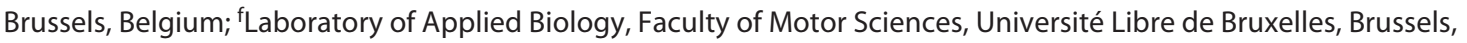 \\ Belgium
}

\section{Keywords \\ Obesity - Exercise - VO2max - Ventilatory threshold · Muscle strength}

\begin{abstract}
Introduction: Patients undergoing weight loss surgery do not improve their aerobic capacity or peak oxygen uptake $\left(\mathrm{VO}_{2}\right.$ peak) after bariatric surgery and some still complain about asthenia and/or breathlessness. We investigated the hypothesis that a post-surgery muscular limitation could impact the ventilatory response to exercise by evaluating the post-surgery changes in muscle mass, strength, and muscular aerobic capacity, measured by the first ventilatory threshold (VT). Methods: Thirteen patients with obesity were referred to our university exercise laboratory before and 6 months after bariatric surgery and were matched by sex, age, and height to healthy subjects with normal weight. All subjects underwent a clinical examination, blood sampling, and body composition assessment by dual-energy X-ray absorptiometry, respiratory and limb muscle strength assessments, and cardiopulmonary exercise testing on a cyclo-ergometer. Results: Bariatric surgery resulted in a loss of $34 \%$ fat mass, $43 \%$ visceral adipose tissue, and 12\% lean mass (LM) ( $p<$
\end{abstract}

(C) 2021 The Author(s)

Published by S. Karger AG, Basel

This is an Open Access article licensed under the Creative Commons Attribution-NonCommercial-4.0 International License (CC BY-NC) (http://www.karger.com/Services/OpenAccessLicense), applicable to the online version of the article only. Usage and distribution for commercial purposes requires written permission.
0.001). Absolute handgrip, quadriceps, or respiratory muscle strength remained unaffected, while quadriceps/handgrip strength relative to LM increased $(p<0.05)$. Absolute $\mathrm{VO}_{2}$ peak or $\mathrm{VO}_{2}$ peak/LM did not improve and the first VT was decreased after surgery $\left(1.4 \pm 0.3\right.$ vs. $1.1 \pm 0.4 \mathrm{~L} \mathrm{~min}^{-1}, p<$ $0.05)$ and correlated to the exercising LM (LM legs) $(R=0.84$, $p<0.001)$. Conclusions: Although bariatric surgery has numerous beneficial effects, absolute $\mathrm{VO}_{2}$ peak does not improve and the weight loss-induced LM reduction is associated to an altered muscular aerobic capacity, as reflected by an early VT triggering early exercise hyperventilation.

(c) 2021 The Author(s).

Published by S. Karger AG, Basel

\section{Introduction}

Bariatric surgery is considered as a long-term effective therapy reducing morbidity and mortality [1], while associated with a rapid and significant weight loss, especially in the first 6 months [2]. Previous studies reported beneficial effects of bariatric surgery on physical functioning and mobility: reduced disability, joint pain, arthritis and enhanced musculoskeletal function, walking capacity, exercise economy, endurance test duration, etc. 
[3-5]. Fat mass loss after bariatric surgery also leads to an increased peak oxygen uptake $\left(\mathrm{VO}_{2}\right.$ peak) relative to body weight (expressed in $\mathrm{mL} \mathrm{kg}^{-1} \mathrm{~min}^{-1}$ ), which presents a great advantage for body weight-bearing efforts [6]. However, no study reported an increase in absolute $\mathrm{VO}_{2}$ peak (L/min), reflecting the intrinsic aerobic capacity [6]. Previous studies performed 6 months after surgery reported either a significant absolute $\mathrm{VO}_{2}$ peak drop $[3,7-10]$ or a tendency to decrease [11]. Thus, even when surgery is successful, absolute $\mathrm{VO}_{2}$ peak is not improved and some patients still complain about asthenia or/and breathlessness [6]. The underlying mechanisms of those physical deconditioning symptomatology remain unclear. It has previously been suggested but not conclusively demonstrated that the muscle mass loss accompanying post-surgery weight loss may interfere with aerobic exercise performance after bariatric surgery $[6,12]$. Indeed, the largescale weight loss after bariatric surgery results not only in a substantial loss of fat mass (FM) but is also associated with significant lean mass (LM) loss, with muscle proteins serving as a source of amino acids for the functioning of other cells. Previous studies underlined the deleterious effects of excessive LM loss during weight loss programs on metabolism, thermoregulation, and functional capacity [13]. However, the link between post-bariatric surgery LM loss and the respiratory and cardiovascular response to maximal and submaximal aerobic exercise have been understudied until now. We, therefore, hypothesized that combining accurate body composition assessment (including leg LM), limbs, and respiratory muscle strength measurements, together with a cyclo-ergometer cardio-pulmonary exercise test (CPET), would allow us to describe the physiological influence of a muscle mass limitation on respiratory, cardiovascular, or metabolic response to exercise before and after bariatric surgery. We also believed that comparison with a control group of healthy subjects with normal weight matched for age, sex, and height would highlight the impact of overweight before bariatric surgery and enable to assess whether differences persist 6 months after weight loss surgery.

\section{Methods}

\section{Study Population}

Thirteen patients with obesity ( 6 men/7 women, $49 \pm 14$ years old, $168 \pm 7 \mathrm{~cm}, 112 \pm 17 \mathrm{~kg}$, body mass index [BMI]: $39.5 \pm 3.5 \mathrm{~kg}$ $\mathrm{m}^{-2}$ ) recruited from the local University Hospital, Department of Gastric Surgery performed identical experimental protocol on two occasions: before and 6 months after bariatric surgery. Each pa- tient with obesity included in the present study was individually paired to a healthy subject with normal weight matched by race, sex, age, and height recruited in his social environment. The characteristics of all 26 participants are shown in Table 1.

All participants gave their informed written consent to the study, approved by the local Ethical Committee (reference: P2016/448). Patients suffering from heavy musculoskeletal, cardiovascular, or pulmonary disease or under beta blockers were excluded. However, 5 patients with obesity suffered from mild arterial hypertension, 5 from sleep-apnea, 3 from impaired glucose tolerance, and 7 dyslipidemia. All patients with obesity underwent bariatric surgery without complications: sleeve gastrectomy $(n=12)$ or laparoscopic Roux-en-Y gastric bypass $(n=1)$. After bariatric surgery, all patients benefited from a nutritional follow-up with appointments every 2 months with the same dietitian. All patients were counseled about the principles of healthy eating, with 3 small meals per day, at least 5 daily servings of fresh fruits and vegetables and vitamin supplements. Protein intake recommendations were individualized regarding sex, age, and weight. A minimal protein intake of $60 \mathrm{~g} /$ day and up to $1.5 \mathrm{~g} / \mathrm{kg}$ ideal weight per day was targeted.

The patients were advised to increase their daily physical activities and to walk as much as possible. However, no controlled or structured exercise training was imposed or proposed.

Healthy subjects with normal weight declared themselves as healthy and free from any proven pathology. All participants had a normal electrocardiogram (ECG) at rest.

\section{Experimental Protocol}

All subjects were invited to the laboratory to perform the following test sequence: clinical examination and fasting blood sampling, body composition assessment, respiratory and skeletal muscle strength assessment followed by a CPET. Patients with obesity repeated the protocol 6 months after bariatric surgery.

\section{Clinical Assessment}

Clinical assessment included a medical history, clinical examination with measurements of resting blood pressure (sphygmomanometry), ECG, pulsed oximetry (Nelcor Puritan Bennett Inc., Pleasanton, CA, USA), and fasting blood sampling. Fasting blood tests were analyzed for metabolic syndrome assessment by the same hospital laboratory with measurements of fasting glycemia, triglyceride, and high-density lipoprotein cholesterol. The hemoglobin level was also evaluated, being a determinant of $\mathrm{O}_{2}$ transport and a potential limiting factor of the $\mathrm{VO}_{2}$ peak.

The Global Physical Activity Questionnaire (GPAQ) was used to assess self-estimated moderate and vigorous intensity activities amount and self-estimated sedentary behavior [14]. The questionnaires were completed by the investigator during an interview with each of the participants.

\section{Anthropometry}

All the measurements were performed in the morning after an overnight fast. Stature was measured to the nearest $0.5 \mathrm{~cm}$ with a wall-mounted stadiometer. Weight was measured to the nearest $0.1 \mathrm{~kg}$ on a standing weighting scale (BC-418; TANITA, Tokyo, Japan) wearing no shoes and light clothing. BMI was expressed in $\mathrm{kg} / \mathrm{m}^{2}$ where $\mathrm{kg}$ is the person's weight in kilograms and $\mathrm{m}^{2}$ is the height in meters squared. The percentage of excess of BMI loss after surgery was calculated based on an ideal BMI of $25 \mathrm{~kg} / \mathrm{m}^{2}$. Waist circumference was assessed with a standard flexible nonelas- 
Table 1. Participant's anthropometric, body composition, metabolic, physical activity characteristics

\begin{tabular}{|c|c|c|c|}
\hline & Pre-surgery & Post-surgery & Controls \\
\hline Men/women ratio & $6 / 7$ & $6 / 7$ & $6 / 7$ \\
\hline Age, years & $49 \pm 14$ & $49 \pm 14$ & $48 \pm 13$ \\
\hline Height, m & $1.68 \pm 0.07$ & $1.68 \pm 0.07$ & $1.73 \pm 0.07$ \\
\hline Weight, kg & $112 \pm 17$ & $87 \pm 16^{\$ \$ \$}$ & $70 \pm 11^{\circ 00, * *}$ \\
\hline $\mathrm{BMI}, \mathrm{kg} \mathrm{m}^{-2}$ & $39.5 \pm 3.5$ & $30.7 \pm 3.4^{\$ \$ \$}$ & $23.3 \pm 3.1^{000, * * *}$ \\
\hline Total body FM, kg & $53 \pm 9$ & $36 \pm 10^{\$ \$ \$}$ & $19 \pm 6^{\circ 00, * *}$ \\
\hline Percentage body fat, $\%$ & $49 \pm 4$ & $41 \pm 5^{\$ \$ \$}$ & $31 \pm 7^{000, * * *}$ \\
\hline VAT, $g$ & $2,685 \pm 1,329$ & $1,490 \pm 750^{\$ \$ \$}$ & $660 \pm 743^{\circ \circ \circ, *}$ \\
\hline Trunk FM, kg & $29 \pm 4$ & $19 \pm 4^{\$ \$ \$}$ & $9 \pm 3^{\circ \circ 0}, * * *$ \\
\hline Arms FM, kg & $5 \pm 1$ & $4 \pm 1^{\$ \$ \$}$ & $2 \pm 0^{\circ 00, * * *}$ \\
\hline Legs FM, kg & $18 \pm 6$ & $12 \pm 5^{\$ \$ \$}$ & $8 \pm 2^{\circ 00, * *}$ \\
\hline Total body LM, kg & $56 \pm 10$ & $49 \pm 8^{\$ \$ \$}$ & $46 \pm 7^{\circ}$ \\
\hline Trunk LM, kg & $25 \pm 4$ & $23 \pm 4^{\$ \$ \$}$ & $21 \pm 4^{\circ \circ}$ \\
\hline Arms LM, kg & $6 \pm 1$ & $5 \pm 1^{\$ \$ \$}$ & $5 \pm 2^{\circ 00}$ \\
\hline Legs LM, kg & $21 \pm 4$ & $18 \pm 3^{\$ \$ \$}$ & $18 \pm 4^{\circ \circ}$ \\
\hline Hemoglobin, $\mathrm{mg} \mathrm{dL}^{-1}$ & $13.4 \pm 1.7$ & $13.8 \pm 1.1$ & $14.5 \pm 0.9$ \\
\hline Waist circumference, $\mathrm{cm}$ & $127 \pm 9$ & $106 \pm 10^{\$ \$}$ & $85 \pm 10^{\circ 00, * * *}$ \\
\hline Mean $\mathrm{BP}_{\text {rest }}, \mathrm{mm} \mathrm{Hg}$ & $99 \pm 13$ & $84 \pm 13^{\$ \$ \$}$ & $90 \pm 13$ \\
\hline Fasting glycemia, $\mathrm{mg} \mathrm{dL}^{-1}$ & $107 \pm 30$ & $85 \pm 7^{\$ \$}$ & $93 \pm 10$ \\
\hline Triglyceride, $\mathrm{mg} \mathrm{dL}^{-1}$ & $136 \pm 59$ & $94 \pm 45^{\$}$ & $93 \pm 52$ \\
\hline $\mathrm{HDL}-\mathrm{C}, \mathrm{mg} \mathrm{dL}^{-1}$ & $47 \pm 13$ & $56 \pm 11^{\$}$ & $65 \pm 19^{\circ \circ}$ \\
\hline GPAQ sedentary time, min day $^{-1}$ & $562 \pm 158$ & $576 \pm 180$ & $496 \pm 196$ \\
\hline GPAQ moderate physical activity time, $\min \mathrm{wk}^{-1}$ & $438 \pm 536$ & $627 \pm 584^{\$}$ & $507 \pm 433$ \\
\hline GPAQ vigorous physical activity time, min $\mathrm{wk}^{-1}$ & $0 \pm 0$ & $9 \pm 23$ & $106 \pm 107^{\circ 00, * * *}$ \\
\hline
\end{tabular}

VAT, visceral adipose tissue; BP, blood pressure; HDL-C, high-density lipoprotein cholesterol. ${ }^{\circ} p<0.05,{ }^{\circ 0} p<$ $0.01,{ }^{\circ 00} p<0.001$ : pre-surgery versus controls. ${ }^{*} p<0.05,{ }^{* *} p<0.01,{ }^{* * *} p<0.001$ : post-surgery versus controls. ${ }_{\$} p<0.05,{ }^{\$ \$} p<0.001$ : pre-surgery versus post-surgery.

tic metric tape over the midpoint between the last rib and the iliac crest with the patient standing and exhaling. Total and regional FM and LM were acquired using dual energy X-ray absorptiometry (Lunar Prodigy; GE Healthcare, Madison, WI, USA) and analyzed using enCORE software (version 15.0). The regions of interest (ROI) for regional body composition (left and right arm, left and right leg, trunk) were first defined automatically by the software. Then the arm and leg ROI were manually corrected by the investigator to make them cut proximally across the coracoid process and the line along the lower ramus and the opening of the acetabulum, respectively [15]. Visceral adipose tissue analysis was performed using fully automated software (CoreScan; GE Healthcare). The software segments the abdominal fat measured by dual energy X-ray absorptiometry into subcutaneous fat and visceral fat within the android region of the abdomen. VAT is then estimated by subtracting subcutaneous fat from the total android fat. The method has previously been validated against computed tomography in patients with a wide range of BMI $[16,17]$.

\section{Muscle Strength Measurements}

Isometric quadriceps strength was measured during knee extension using a digital force gauge (Sauter FK 1K, Balingen, Germany) in a sitting position, knee angle at $90^{\circ}$. Relative quadriceps strength was expressed as the ratio of absolute quadriceps strength divided by the lower limb LM.
During handgrip muscle strength evaluation, subjects were asked to squeeze a dynamometer as hard as possible (Idass Fitness, Cornwall, UK) in a standardized standing position, with a $90^{\circ}$ shoulder flexion and complete elbow extension. Relative handgrip strength was expressed as the ratio of absolute handgrip strength divided by the upper limb LM.

A respiratory pressure meter (Micro RPM; CareFusion, Wokingham, UK) was used to assess the respiratory muscle strength. This unit measured the maximum inspiratory pressure (MIP) and expiratory pressure (MEP). The device was connected to a PC running PUMA (MicroRPM) allowing real-time display of respiratory pressure. Measurements were performed according to ATS/ ERS statements for respiratory muscle testing at rest, in the seated position with a nasal clip [18]. MIP was determined as the maximal pressure recorded during an inspiration maneuver starting from residual lung volume. MEP was determined as the maximal pressure recorded during an expiration maneuver starting from total lung capacity. Muscle strength measurements were repeated at least five times, with minimum three reproducible maneuvers (variation equal or less than 10\%) and the highest value was considered for analyses.

\section{CPET}

Aerobic capacity was assessed using a classical incremental CPET on an electrically braked cyclo-ergometer (Ergoselect II 
1200; Ergoline, Bitz, Germany). $\mathrm{VO}_{2}, \mathrm{CO}_{2}$ production $\left(\mathrm{VCO}_{2}\right)$, and ventilation (VE) were collected breath by breath through a facial mask and analyzed every $8 \mathrm{~s}$ using a metabolic system (Exp'Air ${ }^{\circledR}$; Medisoft, Dinant, Belgium) calibrated with room air and standardized gas. Expiratory volume in $1 \mathrm{~s}$ was measured at rest before the exercise test to calculate the maximum ventilatory ventilation.

The CPET was performed in agreement with ERS guidelines [19]. The initial power started at $30 \mathrm{~W}$ for warm up with increments of $15-30 \mathrm{~W} / \mathrm{min}$, estimated from previous CPET performance and for an optimal test duration between 10 and 12 min until volitional exhaustion. Identical incremental CPET workload protocol was repeated before and after bariatric surgery and imposed to the matched control subject. Heart rate (HR), ECG, and pulsed oximetry were continuously monitored during the test. Effort was considered maximal when two of the following criteria were met: $\mathrm{VO}_{2}$ increase less than $100 \mathrm{~mL} / \mathrm{min}$ while workload further increases, respiratory exchange ratio (RER) $>1.10$, achievement of age predicted maximal $\mathrm{HR}$, and incapacity to maintain the pedal rate $\geq 50 \mathrm{rpm}$. $\mathrm{VO}_{2}$ peak was expressed in absolute value, relative to body weight or relative to LM. The VT, used as a surrogate of muscle aerobic exercise capacity, was determined by the $\mathrm{V}$-slope method by two blinded independent experienced exercise physiologists. Chemosensibility and ventilatory efficiency were assessed using the $\mathrm{VE} / \mathrm{VCO}_{2}$ slope measured during the entire exercise test. The $\mathrm{VE} / \mathrm{VO}_{2}$ ratio was reported to evaluate the ventilatory cost for a given $\mathrm{O}_{2}$ metabolism. The $\mathrm{HR} / \mathrm{VO}_{2}$ slope measured throughout the test was used to quantify the chronotropic response to exercise. The metabolic efficiency during exercise was evaluated by calculation of the $\mathrm{VO}_{2}$ /workload (W) slope measured from rest to the respiratory compensation point. $\mathrm{O}_{2}$ pulse and VT were corrected by the LM of the lower limbs (LM legs) as it reflects the main muscle mass consuming $\mathrm{O}_{2}$ during a cycloergometer exercise. This correction was used to dissociate the convective (stroke volume [SV] or cardiac output [Q]) and the muscular $\mathrm{O}_{2}$ extraction $\left(\mathrm{Ca}-\mathrm{vO}_{2}\right)$ component of $\mathrm{O}_{2}$ pulse and VT, respectively.

\section{Statistical Analysis}

Data are presented as a mean \pm SD. Normal distribution of the data was tested using the Shapiro-Wilk test. Normally distributed data were compared using unpaired $t$ test for the comparison of the pre-surgery condition versus control group, and post-surgery condition versus control group, and paired $t$ test was used for the pre- versus post-surgery conditions comparisons. Pearson's correlation coefficient was used for the analysis of associations between VT and LM in the different groups. Data analysis was conducted using GraphPad Prism 8 (GraphPad Software, San Diego, CA, USA) and significancy threshold was set at a $p$ value lower than 0.05 .

\section{Results}

\section{Body Composition}

Six months after bariatric surgery, all these parameters were significantly decreased (Table 1) with a mean total body weight loss of $22 \%(-25 \pm 8 \mathrm{~kg})$, which repre-

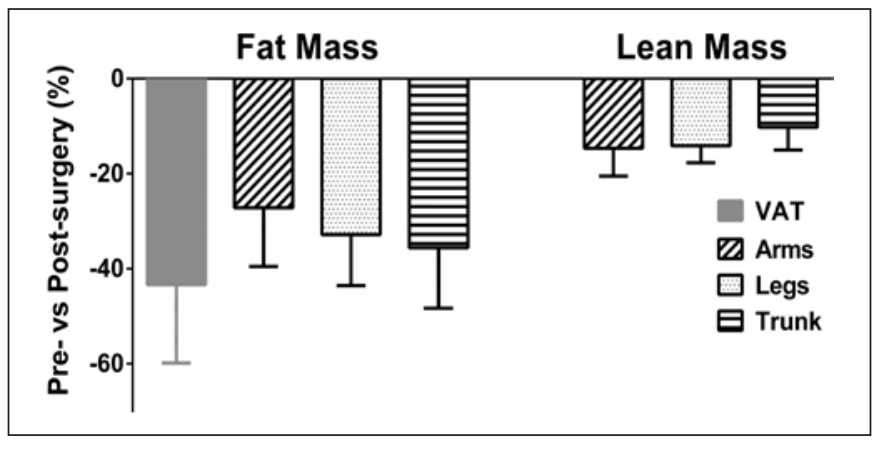

Fig. 1. Regional changes in FM and LM 6 months after bariatric surgery. All the reported changes pre- versus post-surgery were statistically significant $(p<0.001)$. VAT reduction exhibited the most important change compared to any other regional fat mass loss $(p<0.001)$. Arms FM loss was lower than legs and trunk FM loss $(p<0.05)$ which were not different $(p>0.05)$. LM loss was homogenously reduced over the different body regions $(p>0.05)$. VAT, visceral adipose tissue.

sents a change in BMI of $-8.8 \pm 2.8 \mathrm{~kg} / \mathrm{m}^{2}$ and an excess of BMI loss of $-23 \pm 7 \%$. The FM loss induced by bariatric surgery $(-34 \pm 11 \%)$ was associated to a decrease in LM $(-12 \pm 3 \%)$. Regional post-surgery changes in FM and LM are shown in Figure 1. For FM, the greatest change was observed for VAT $(-43 \pm 16 \%)$, while LM was homogeneous reduced in all body regions after surgery and was no more significantly different from healthy subjects with normal weight. Six months after surgery, VAT, total FM, and relative FM (\%) remained significantly higher in patients as compared to healthy subjects with normal weight.

\section{Metabolic Characteristics}

Bariatric surgery improved metabolic parameters (mean blood pressure, high-density lipoprotein cholesterol, triglyceride, fasting glycemia) which were no more different from healthy subjects with normal weight. $\mathrm{Hb}$ levels remained unaffected by surgery (Table 1).

\section{Daily Physical Activity Level}

The results of the GPAQ questionnaire are exposed in Table 1. After bariatric surgery, self-estimated sedentary, and vigorous physical activity time remained unchanged and moderate activity time was slightly improved. No difference in daily sedentary time and weekly moderate activity time were observable when comparing post-surgery patients with obesity versus healthy subjects with normal weight but vigorous physical activity time remained lower in patients. 
Table 2. Respiratory muscle strength and spirometry before and after bariatric surgery versus healthy matched controls

Table 3. CPET before and after bariatric surgery versus healthy matched controls

\begin{tabular}{llll}
\hline & Pre-surgery & Post-surgery & Controls \\
\hline Limb muscle strength & & & \\
Quadriceps strength, kg & $53 \pm 15$ & $51 \pm 13$ & $50 \pm 10$ \\
$\quad$ Quadriceps strength/leg LM & $5.0 \pm 1.1$ & $5.5 \pm 0.8^{\$}$ & $5.7 \pm 0.9^{\circ}$ \\
$\quad$ Handgrip strength, kg & $36 \pm 9$ & $37 \pm 9$ & $38 \pm 9$ \\
$\quad$ Handgrip strength/arm LM & $11.4 \pm 2.0$ & $13.7 \pm 3.0^{\$ \$}$ & $15.2 \pm 1.4^{\circ \circ 0}$ \\
Respiratory muscle strength, $\mathrm{cm} \mathrm{H} \mathrm{H}_{2} \mathrm{O}$ & & & \\
$\quad$ MIP & $90 \pm 18$ & $93 \pm 23$ & $85 \pm 22$ \\
MEP & $116 \pm 28$ & $114 \pm 23$ & $115 \pm 40$ \\
\hline
\end{tabular}

${ }^{\circ} p \leq 0.05,{ }^{\circ 00} p<0.001$ : pre-surgery versus controls. ${ }^{\$} p<0.05,{ }^{\$ \$} p<0.01$ : pre-surgery versus post-surgery.

\begin{tabular}{|c|c|c|c|}
\hline & Pre-surgery & Post-surgery & Controls \\
\hline Workload $_{\max }$, Watt & $124 \pm 32$ & $135 \pm 44^{\$}$ & $167 \pm 34^{\circ \circ}$ \\
\hline $\mathrm{VO}_{2}$ peak, $\mathrm{L} \mathrm{min}^{-1}$ & $1.9 \pm 0.5$ & $1.8 \pm 0.5$ & $2.1 \pm 0.5^{* * *}$ \\
\hline $\mathrm{RER}_{\max }$ & $1.07 \pm 0.10$ & $1.25 \pm 0.13^{\$ \$}$ & $1.21 \pm 0.15^{\circ}$ \\
\hline $\mathrm{HR}_{\max }, \mathrm{bpm}$ & $143 \pm 18$ & $147 \pm 22$ & $161 \pm 16^{\circ \circ}$ \\
\hline $\mathrm{VE}_{\max }, \mathrm{L} \min ^{-1}$ & $70 \pm 20$ & $78 \pm 28$ & $82 \pm 20$ \\
\hline $\mathrm{VE}_{\max } / \mathrm{MVV}, \%$ & $71 \pm 16$ & $72 \pm 20$ & $65 \pm 22$ \\
\hline $\mathrm{VE} / \mathrm{VO}_{2}$ peak & $37 \pm 7$ & $44 \pm 8^{\$ \$ \$}$ & $40 \pm 7$ \\
\hline $\mathrm{O}_{2}$ pulse $\max , \mathrm{mLO}_{2}$ beat $^{-1}$ & $13.7 \pm 2.7$ & $12.1 \pm 3.4$ & $14.2 \pm 4.1$ \\
\hline $\mathrm{O}_{2}$ pulse $\max / \mathrm{LM}_{\text {legs }}, \mathrm{mLO}_{2}$ beat $^{-1} \mathrm{~kg}$ & $0.64 \pm 0.1$ & $0.66 \pm 0.1$ & $0.83 \pm 0.2^{\circ \circ}$ \\
\hline Maximal $\mathrm{SpO}_{2}, \%$ & $96 \pm 2.8$ & $96 \pm 1.2$ & $94 \pm 5$ \\
\hline $\mathrm{VO}_{2} @ \mathrm{VT}, \mathrm{L} \mathrm{min}^{-1}$ & $1.4 \pm 0.3$ & $1.1 \pm 0.4^{\$}$ & $1.4 \pm 0.4^{* * *}$ \\
\hline $\mathrm{VO}_{2} / \mathrm{LM}_{\text {legs }} @ \mathrm{VT}, \mathrm{mL} \mathrm{kg}{ }^{-1} \mathrm{~min}^{-1}$ & $64 \pm 14$ & $59 \pm 13$ & $80 \pm 18^{\circ}, * *$ \\
\hline VE/ $\mathrm{VCO}_{2}$ slope & $33 \pm 6$ & $33 \pm 6$ & $32 \pm 4$ \\
\hline $\mathrm{VO}_{2} / \mathrm{W}$ slope, $\mathrm{L} \mathrm{min}^{-1} \mathrm{~W}^{-1}$ & $10.8 \pm 1.9$ & $9.9 \pm 2.5$ & $10.0 \pm 2.0$ \\
\hline $\mathrm{HR} / \mathrm{VO}_{2}$ slope, beat $\mathrm{L}^{-1}$ & $40 \pm 14$ & $52 \pm 15^{\$}$ & $48 \pm 14$ \\
\hline $\mathrm{HR} / \mathrm{VO}_{2}$ slope, beat $\mathrm{L}^{-1} \mathrm{~kg}^{-1}$ & $5.0 \pm 1.2$ & $4.7 \pm 1.5$ & $3.2 \pm 1.0^{\circ 00, *}$ \\
\hline
\end{tabular}

$\mathrm{MVV}$, maximum ventilatory ventilation; $\mathrm{SpO}_{2}$, pulse oxygen saturation; $\mathrm{VCO}_{2}$, carbon dioxide output; $\mathrm{W}$, workload. ${ }^{\circ} p<0.05,{ }^{\circ 0} p<0.01$, ${ }^{\circ 00} p<0.001$ : pre-surgery versus controls. ${ }^{*} p<0.05,{ }^{* *} p<0.01,{ }^{* * *} p<0.001$ : post-surgery versus controls. ${ }^{\$} p<0.05,{ }^{\$ \$} p<0.01,{ }^{\$ \$} p<$ 0.001: pre-surgery versus post-surgery.

\section{Limbs and Respiratory Muscle Strength}

After bariatric surgery, the relative handgrip and quadriceps force was improved and no more different from healthy controls (Table 2). Neither obesity nor bariatric surgery affected inspiratory or expiratory muscle strength (Table 2).

\section{Aerobic Capacity}

CPET results are displayed in Table 3. Bariatric surgery increased maximal workload, RER, $\mathrm{VE} / \mathrm{VO}_{2}, \mathrm{VO}_{2}$ relative to body weight, and $\mathrm{HR} / \mathrm{VO}_{2}$ slope (beat/L) but absolute $\mathrm{VO}_{2}$ at the VT was reduced. The latest is illus- trates in Figure 2 as well as the different ways of expressing $\mathrm{VO}_{2}$ peak before and after bariatric surgery. No change in $\mathrm{VO}_{2}$ relative to body weight, relative to LM or relative to LM legs at the VT was observed (Table 3; Fig. 2).

After bariatric surgery, the maximal $\mathrm{VO}_{2}$ (absolute, relative to body weight or relative to LM) and the VT level were lower than in control subjects. Absolute VT measured during cycling CPET was positively correlated to the LM of the legs, 6 months after bariatric surgery and in control subjects, but not in the pre-surgery condition (Fig. 3). 


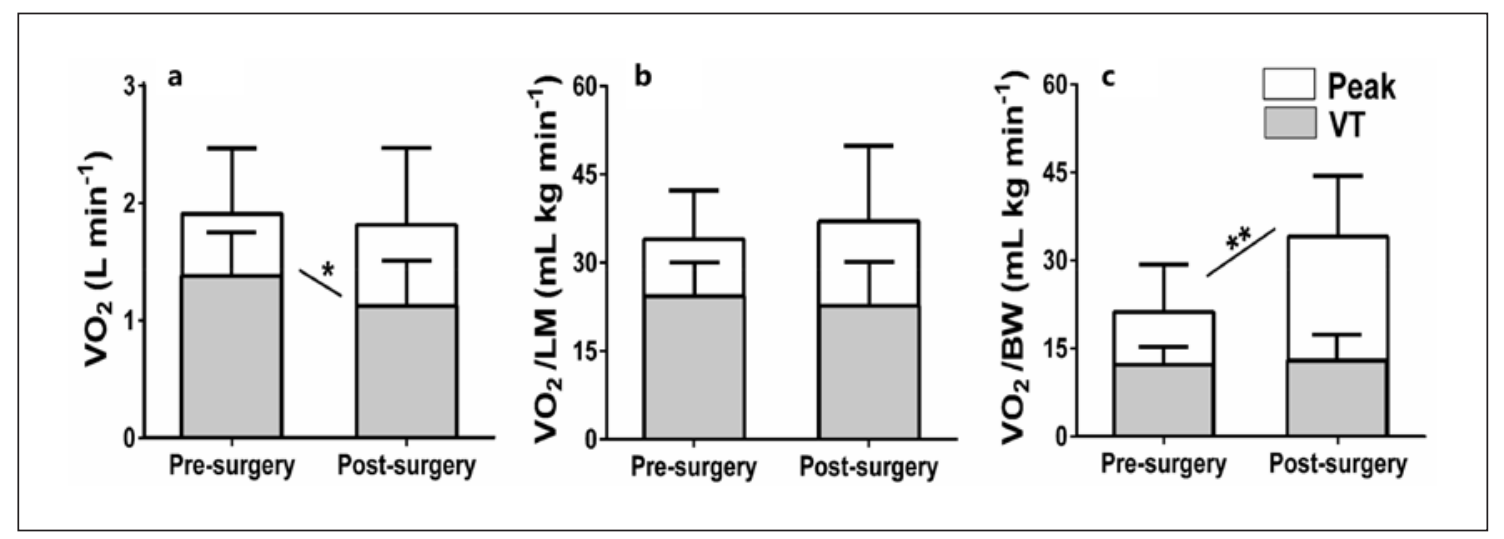

Fig. 2. Comparisons of pre- versus post-surgery $\mathrm{VO}_{2}$ at the $\mathrm{VT}$ (gray) and at peak exercise (white). $\mathrm{VO}_{2}$ is expressed in absolute value (a), adjusted for LM (b), and adjusted for body weight (BW) (c). Six months after bariatric surgery, $\mathrm{VO}_{2}$ peak relative to $\mathrm{BW}$ increased due to weight loss, absolute $\mathrm{VO}_{2}$ peak, and $\mathrm{VO}_{2}$ relative to $\mathrm{LM}$ remained unchanged and the absolute VT was reduced. ${ }^{*} p<0.05,{ }^{* *} p<0.01$.

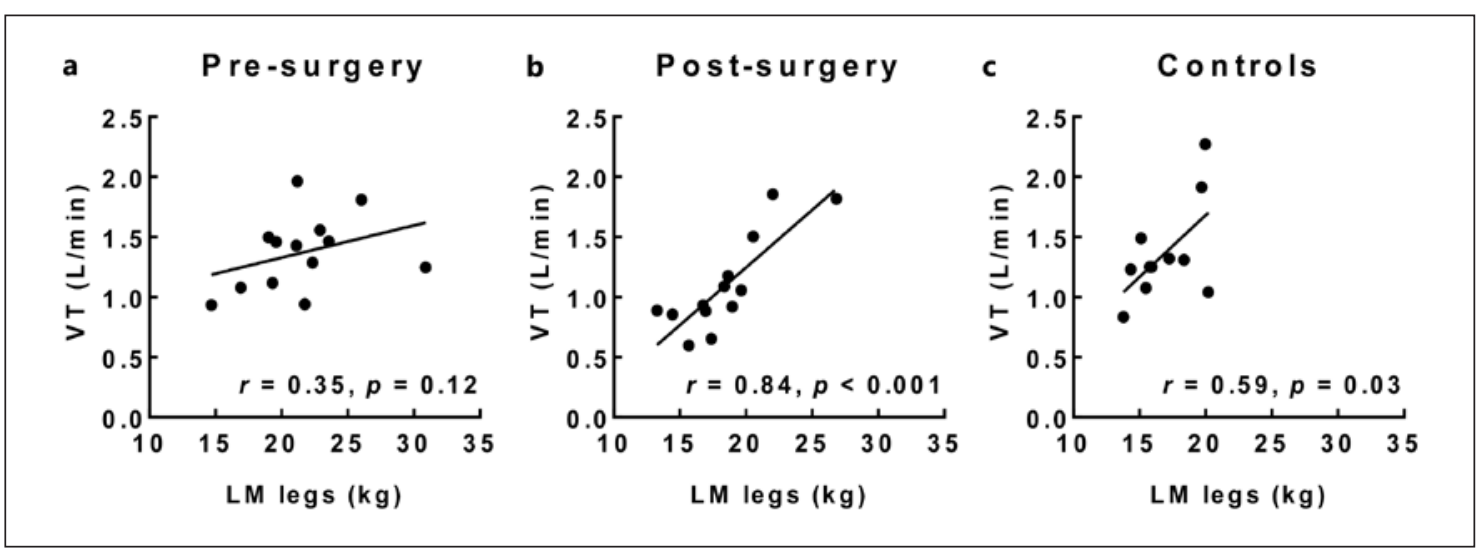

Fig. 3. Absolute $\mathrm{VO}_{2}$ at the first VT as a function of the lower limbs LM (LM legs) in obese patients before bariatric surgery (a), 6 months after bariatric surgery (b) and in healthy control subjects (c). VT was significantly correlated to LM legs after bariatric surgery and in healthy controls.

\section{Discussion}

The present results confirm the positive effects of bariatric surgery on metabolic prognostic factors and the muscle strength/LM ratio. However, 6 months after bariatric surgery, absolute $\mathrm{VO}_{2}$ peak did not improve, and the VT was reduced. This appeared in a context of a $12 \%$ decrease in lean body mass. The presently observed positive correlation between the cycling LM and the VT measured during a cyclo-ergometer CPET after bariatric surgery gives credit to the tested hypothesis of a post-surgery muscular aerobic capacity limitation impacting the ventilatory response to exercise, characterized by early an- aerobic metabolism and early hyperventilation stimulations.

While patients with obesity are known to have impaired skeletal muscle aerobic function, previous studies reported that bariatric surgery may have beneficial qualitative muscular effects on oxidative capacity but associated to a quantitative muscular alteration [20,21]. Indeed, during the heavy weight loss phase, proteolysis provides a source of amino acids needed for metabolic cell functions causing muscle mass reduction [13]. The present study reported a 12\% LM loss 6 months after surgery with the legs LM after weight loss positively correlated to the $\mathrm{VO}_{2}$ at the VT (Fig. 3b). This suggests that even if weight loss 
may have beneficial effects on aerobic muscle function, a lower muscle mass after surgery overrides the positive qualitative effects and is associated to an overall lower muscular aerobic capacity, as reflected by lower VT. This is of importance as VT represents the energy requirement level above which anaerobic metabolism is activated and is associated with a consequent hyperventilation response $[22,23]$. Interestingly, a low VT associated to a high maximal RER is typically observed in muscular deconditioning conditions with early triggering of the anaerobic metabolism activation and early hyperventilation response [24]. As the VE-breathlessness relationship during exercise has been shown to remain unaffected after bariatric surgery, one can suspect that this early exercise induced hyperventilation may cause respiratory discomfort at lower submaximal exercise intensities [25].

\section{Cardiovascular Response to Exercise}

In the present study, weight loss surgery stimulated the chronotropic response as reflected by the increase of the $\mathrm{HR}$ versus absolute $\mathrm{VO}_{2}$ slope. Neunhaeuserer et al. [3] suggested that since cardiovascular and pulmonary function are not supposed to be negatively affected by bariatric surgery, an increased chronotropic response rather reflects a cardiac compensatory effect of a decreased muscular aerobic capacity.

Maximal $\mathrm{O}_{2}$ pulse, the product of maximal stroke volume $\left(\mathrm{SV}_{\max }\right)$ and peak arteriovenous oxygen content difference (Ca- $\mathrm{vO}_{2}$ peak), was previously found to be unchanged or declined after surgery, in relation to an altered muscular oxygen extraction ( $\mathrm{Ca}-\mathrm{vO}_{2}$ peak) [6]. Maximal $\mathrm{O}_{2}$ pulse corrected for the exercising muscle mass (LM legs) better reflects $\mathrm{SV}_{\max }$ when comparing situations of quantitative muscle mass changes (cfr post-surgery reduction of LM). This indirect index of $S V_{\text {max }}$ remained unaffected after surgery in the present study, suggesting little or no influence of weight loss on $\mathrm{SV}_{\max }$. Therefore, according to Fick's Principle, it might be speculated that identical absolute $\mathrm{VO}_{2}$ peak after surgery was related to an unchanged $\mathrm{SV}_{\text {max }}$ and a compensatory chronotropic stimulation in response to a lower $\mathrm{Ca}-\mathrm{vO}_{2}$ induced by lower limb muscle mass loss.

\section{Respiratory Response to Exercise}

Previous studies reported that VAT and subcutaneous trunk fat loss after bariatric surgery improves overall pulmonary function with reduced work of breathing an enhanced ventilatory response to exercise resulting in improved gas exchange $[26,27]$. In the present study, subjects suffering from obesity showed no ventilatory efficiency or chemosensibility alteration with no ventila- tory limitation or exercise induced hypoxemia. However, post-bariatric surgery increase in maximal VE $/ \mathrm{VO}_{2}$ might reflect a muscular deconditioning state with high hyperventilatory response to exercise.

\section{Muscle Strength}

The present results show no difference in MIP and MEP between the pre- and post-surgery conditions and compared to healthy subjects with normal weight. Previous studies showed conflicting results with either unchanged [28], increased [29], or reduced [30] respiratory muscle strength 6 months after bariatric surgery. Type II errors, male/female equilibrium, initial BMI, FM distribution, and loss may account for discrepancies between studies.

The increase in relative (but not absolute) limb muscle strength after the bariatric surgery, among others mechanisms, could be related to an improvement in both muscle activation (triggered by the increased moderate physical activity time) and/or alleviation of central obesity (VAT loss), known to affect muscular metabolism pathways and inflammation [31].

\section{Limitations}

The number of patients evaluated before and after bariatric surgery was small and thus type II but also type I errors could have occurred in spite of a paired sample design or a careful matching. Moreover, physical activity levels were assessed using a physical activity questionnaire which presents a lower reliability as compared to objective methods [32]. While GPAQ validity may be criticized, a recent systematic review by Keating et al. [32] showed a good to very good reliability for quantifying time spent in moderate and vigorous activities. To improve GPAQ validity, we used the interviewer-administered version of the questionnaire since it presents a higher correlation with accelerometer measurements for moderate-to-vigorous physical activity than the self-administrated version [33]. It should also be emphasized that both nutritional status and physical activity levels highly influence the post-bariatric surgery LM loss and may therefore have influenced the present results. The adherence to the protein intake recommendations may interfere with LM loss but was not measured in the present study.

\section{Conclusion}

Bariatric surgery reduced total FM and VAT with considerable positive metabolic benefits associated with a preserved cardiovascular response to exercise resulting in 
unchanged aerobic capacity. However, bariatric surgery results in a substantial LM loss which is associated with a decreased VT. Indeed, LM is correlated to the VT suggesting that a lower LM alters the muscular aerobic capacity after bariatric surgery. Consequently, a slightly increased chronotropic response to exercise and an early triggering of exercise hyperventilation response are observed. The present results support the idea that a muscular aerobic capacity limitation should be taken into consideration during bariatric surgery follow-up in particular when LM loss, low VT, or exercise breathlessness complaints are observed.

\section{Acknowledgments}

We would like to thank all the participants of the study. We express our gratitude to the Department of Surgery of Erasmus Hospital for their sincere cooperation. We offer special thanks to Michel Lamotte (PhD, Physiotherapist), Godefroid Chantal (Physiotherapist), Marie Barea (Nutritionist), and Alain Carpentier for their help and encouragement.

\section{Statement of Ethics}

All procedures performed in studies involving human participants were in accordance with the ethical standards of the institutional and/or national research committee and with the $1964 \mathrm{Hel}-$ sinki Declaration and its later amendments or comparable ethical standards. Written informed consent was obtained from all individual participants included in the study, approved by the local Ethical Committee (reference: P2016/448).

\section{Conflict of Interest Statement}

The authors have no conflicts of interest to declare.

\section{Funding Sources}

The authors received no funding for this work.

\section{Author Contributions}

Concept and design: N.Z., V.F., P.L., J.C., and J.-J.M. Data acquisition and Investigation: N.Z., C.S., K.F., and M.K. Data analysis and interpretation: N.Z., C.S., V.F., K.F., M.K., and G.D. Writing - original draft: N.Z. and V.F. Review and editing: N.Z., M.K., K.F., G.D., J.-J.M., J.C., and P.L. Coordinator - overall and concrete affairs: N.Z.

\section{Data Availability Statement}

The datasets used and/or analyzed during the current study are available from the corresponding author on reasonable request.

\section{References}

1 Colquitt JL, Pickett K, Loveman E, Frampton GK. Surgery for weight loss in adults. Cochrane Database Syst Rev. 2014 Aug;8: CD003641.

2 Tamboli RA, Hossain HA, Marks PA, Eckhauser AW, Rathmacher JA, Phillips SE, et al. Body composition and energy metabolism following Roux-en-Y gastric bypass surgery. Obesity. 2010 Sep;18(9):1718-24.

3 Neunhaeuserer D, Gasperetti A, Savalla F, Gobbo S, Bullo V, Bergamin M, et al. Functional evaluation in obese patients before and after sleeve gastrectomy. Obes Surg. 2017; 27(12):3230-9.

4 Hansen D, Decroix L, Devos Y, Nocca D, Cornelissen V, Dillemans B, et al. Towards optimized care after bariatric surgery by physical activity and exercise intervention: a review. Obes Surg. 2020 Mar;30(3):1118-25.

5 Berglind D, Willmer M, Eriksson U, Thorell A, Sundbom M, Uddén J, et al. Longitudinal assessment of physical activity in women undergoing Roux-en-Y gastric bypass. Obes Surg. 2015 Jan;25(1):119-25.

6 Dereppe H, Forton K, Pauwen NY, Faoro V. Impact of bariatric surgery on women aerobic exercise capacity. Obes Surg. 2019;29(10): 3316-23.

7 Lund MT, Hansen M, Wimmelmann CL, Taudorf LR, Helge JW, Mortensen E, et al. Increased post-operative cardiopulmonary fitness in gastric bypass patients is explained by weight loss. Scand J Med Sci Sports. 2016 Dec; 26(12):1428-34.

8 Kanoupakis E, Michaloudis D, Fraidakis O, Parthenakis F, Vardas P, Melissas J. Left ventricular function and cardiopulmonary performance following surgical treatment of morbid obesity. Obes Surg. 2001 Oct;11(5):552-8.

9 Nedeljkovic-Arsenovic O, Banovic M, Radenkovic D, Rancic N, Polovina S, Micic D, et al. The amount of weight loss six months after bariatric surgery: it makes a difference. Obes Facts. 2019 Jul;12(3):281-90.

10 Bellicha A, Ciangura C, Roda C, Torcivia A, Portero P, Oppert J-M. Changes in cardiorespiratory fitness after gastric bypass: relations with accelerometry-assessed physical activity. Obes Surg. 2019;29(9):2936-41.

11 Onofre T, Carlos R, Oliver N, Felismino A, Fialho D, Corte R, et al. Effects of a physical activity program on cardiorespiratory fitness and pulmonary function in obese women after bariatric surgery: a Pilot Study. Obes Surg. 2017;27(8):2026-33.

12 Wilms B, Ernst B, Thurnheer M, Weisser B, Schultes B. Differential changes in exercise performance after massive weight loss induced by bariatric surgery. Obes Surg. 2013; 23(3):365-71.

13 Marks BL, Rippe JM. The importance of fat free mass maintenance in weight loss programmes. Sports Med. 1996;22(5):273-81.

14 Rivière F, Widad FZ, Speyer E, Erpelding ML, Escalon H, Vuillemin A. Reliability and validity of the French version of the global physical activity questionnaire. J Sport Health Sci. 2018;7(3):339-45.

15 Lohman M, Tallroth K, Kettunen JA, Marttinen MT. Reproducibility of dual-energy $\mathrm{x}$ ray absorptiometry total and regional body composition measurements using different scanning positions and definitions of regions. Metabolism. 2009;58(11):1663-8.

16 Kaul S, Rothney MP, Peters DM, Wacker WK, Davis CE, Shapiro MD, et al. Dual-energy Xray absorptiometry for quantification of visceral fat. Obesity. 2012;20(6):1313-8. 
17 Ergun DL, Rothney MP, Oates MK, Xia Y, Wacker WK, Binkley NC. Visceral adipose tissue quantification using lunar prodigy. J Clin Densitom. 2013;16(1):75-8.

18 American Thoracic Society/European Respiratory Society. ATS/ERS statement on respiratory muscle testing. Am J Respir Crit Care Med. 2002;166(4):518-624.

19 ERS Task Force; Palange P, Ward SA, Carlsen $\mathrm{KH}$, Casaburi R, Gallagher CG, et al. Recommendations on the use of exercise testing in clinical practice. Eur Respir J. 2007;29:185209.

20 Bray GA, Bouchard C. The biology of human overfeeding: a systematic review. Obes Rev. 2020;21(9):e13040.

21 Giusti V, Theytaz F, Di Vetta V, Clarisse M, Suter M, Tappy L. Energy and macronutrient intake after gastric bypass for morbid obesity: a 3-y observational study focused on protein consumption. Am J Clin Nutr. 2016;103(1): $18-24$.

22 Wasserman K, Van Kessel AL, Burton GG Interaction of physiological mechanisms during exercise. J Appl Physiol. 1967;22(1):7185.
23 Poole DC, Rossiter HB, Brooks GA, Gladden LB. The anaerobic threshold: $50+$ years of controversy. J Physiol. 2021;599(3):737-67.

24 Ploutz-Snyder LL, Downs M, Goetchius E, Crowell B, English KL, Ploutz-Snyder R, et al. Exercise training mitigates multisystem deconditioning during bed rest. Med Sci Sports Exerc. 2018;50(9):1920-8.

25 Mainra A, Abdallah SJ, Reid RER, Andersen RE, Jensen D. Effect of weight loss via bariatric surgery for class III obesity on exertional breathlessness. Respir Physiol Neurobiol. 2019 Aug;266:130-7.

26 Alsumali A, Al-Hawag A, Bairdain S, Eguale $\mathrm{T}$. The impact of bariatric surgery on pulmonary function: a meta-analysis. Surg Obes Relat Dis. 2018;14(2):225-36.

27 Zavorsky GS, Kim DJ, Christou NV. Compensatory exercise hyperventilation is restored in the morbidly obese after bariatric surgery. Obes Surg. 2008 May;18(5):549-59.

28 Parreira VF, Matos CM, Athayde FT, Moraes KS, Barbosa MH, Britto RR. Evolution of respiratory muscle strength in post-operative gastroplasty. Rev Bras Fisioter. 2012;16(3): 225-30.
29 Weiner P, Waizman J, Weiner M, Rabner M, Magadle R, Zamir D. Influence of excessive weight loss after gastroplasty for morbid obesity on respiratory muscle performance. Thorax. 1998;53(1):39-42.

30 Pouwels S, Kools-Aarts M, Said M, Teijink JA, Smeenk FW, Nienhuijs SW. Effects of bariatric surgery on inspiratory muscle strength. Springerplus. 2015;4(1):322.

31 Savolainen AM, Karmi A, Immonen H, Soinio M, Saunavaara V, Pham T, et al. Physical activity associates with muscle insulin sensitivity postbariatric surgery. Med Sci Sports Exerc. 2019;51(2):278-87.

32 Keating XD, Zhou K, Liu X, Hodges M, Liu J, Guan J, et al. Reliability and concurrent validity of global physical activity questionnaire (GPAQ): a systematic review. Int J Environ Res Public Health. 2019;16(21):4128.

$33 \mathrm{Chu} \mathrm{AH}, \mathrm{Ng} \mathrm{SH}$, Koh D, Müller-Riemenschneider F. Reliability and validity of the selfand interviewer-administered versions of the global physical activity questionnaire (GPAQ). PLoS One. 2015;10(9):e0136944. 